\title{
Analysis of the Incidence and Risk factors of Postoperative Delirium in Patients with Degenerative Cervical Myelopathy
}

Seok Woo Kim ( $\nabla$ spinedrk@gmail.com )

Hallym University Sacred Heart Hospital

Jeong Hwan Lee

Hallym University Sacred Heart Hospital

Min Uk Lee

Hallym University Sacred Heart Hospital

Hyung Min Lee

Hallym University Sacred Heart Hospital

Seung Hun Lee

Hallym University Sacred Heart Hospital

Young Eun Kim

Hallym University Sacred Heart Hospital

\section{Research Article}

Keywords: postoperative delirium (POD), incidence; risk factors, degenerative cervical myelopathy (DCM), laminoplasty, ACDF

Posted Date: December 21st, 2020

DOl: https://doi.org/10.21203/rs.3.rs-125496/v1

License: (c) (i) This work is licensed under a Creative Commons Attribution 4.0 International License.

Read Full License 


\section{Abstract}

Delirium is one of the most common postoperative complications in surgical procedures. Postoperative delirium (POD) may affect normal recovery and outcomes after surgery. Degenerative Cervical myelopathy (DCM), a disease that progresses with age, usually associated with neurological symptoms. This study is designed to analyze the incidence and risk factors that cause post-operative delirium in DCM. The incidence and risk factors of delirium were analyzed in 148 patients aged 60 years or older who underwent surgery for DCM. Among the 148 patients who underwent surgery, 78 received laminoplasty, of whom 19 patients (24\%) experienced delirium; the other 70 patients underwent ACDF, of whom five patients $(7.1 \%)$ experienced delirium. The occurrence of POD in the laminoplasty group was 4.18 times higher than that in the ACDF group. Age, and sodium level were found to be significant risk factors for all 148 patients in a multivariate analysis. In the laminoplasty group, age, and high potassium level were meaningful risk factors in multiple regression analysis. Risk factors that affect the POD were not analyzed in ACDF due to the low $n(=5)$. This study shows that the incidence and risk factors for POD can vary depending on the surgical method employed in patients with DCM.

\section{Introduction}

Postoperative delirium (POD) refers to any acute change in cognition, including fluctuating awareness, deficit attention, memory impairment, disorientation, or other behavioral disorders that occur within 30 days after surgery. ${ }^{1,2}$ The overall complication rate was reported at 13 percent in patients with posterior lumbar fusion according to the data of NIS (National Inpatient Sample). ${ }^{3}$ Delirium is one of the most common postoperative complications (10 77\%) and occurs after various surgical treatments including orthopedic, pelvic, and aortic surgeries. ${ }^{4,5}$ POD worsens surgical outcomes, increases hospitalization period, raises the medical cost, and increases postoperative morbidity and mortality. ${ }^{6}$ There are many causes of delirium. Preoperative cognitive impairment, drug abuse or alcoholism, diabetes, hearing or vision impairment, various types of surgery, excessive bleeding, and acute anemia are known as factors of POD. Older age is the most common cause in relation to orthopedic and cardiac surgeries, as well as anesthesia. ${ }^{7,8}$

A few studies on POD after spinal surgery have been conducted and identified various associated factors, including age, baseline comorbidities, preoperative cognition, neurological diseases, and operation duration. ${ }^{9-12}$ However, these studies included all spinal surgery patients with no consideration for factors related to the prevalence of delirium, whose occurrence varies according to the type and method of spinal surgery. Thus, these studies provide no descriptions of whether the degree of delirium would vary according to the severity of the disease, the surgical levels, or the specific surgical method. ${ }^{13,14}$

Degenerative Cervical myelopathy (DCM) is the most common cause of non-traumatic spinal cord injury at the age of 55 and older and can occur due to various causes, including ossification of the posterior longitudinal ligament (OPLL), and degenerative disc disease or stenosis. ${ }^{15}$ 
Early surgical treatment is usually recommended because it not only causes clinical manifestations such as clumsiness of hands, abnormal gait, and bowel or bladder dysfunctions, but also can cause irreversible neurological damage to the spinal cord as the lesion progresses. ${ }^{16,17}$ Surgical methods for DCM can vary according to the extent and location of the lesion. ${ }^{18,19}$ Anterior cervical discectomy and fusion (ACDF) or anterior corpectomy and fusion (ACF) can be performed in the anterior approach, and posterior cervical laminoplasty or posterior laminectomy and fusion can be performed in the posterior approach to relieve the compressed spinal cord, improve blood flow in the spinal cord, and prevent nerve damage. The selection of a surgical approach depends on the location and extent of the lesion. Typically, in the presence of lesions extending over three or more segments and in cases where lordosis of the cervical spine is well maintained in myelopathy with cervical stenosis, posterior laminoplasty or posterior laminectomy and fusion are usually recommended. ${ }^{18,19}$

As far as the authors know, no study has yet provided any data on the incidence and risk factors of POD in patients with DCM. The identification of risk factors involved in the incidence of POD will help prevent delirium. Furthermore, active rehabilitation after surgery is extremely important because DCM patients may have poor outcomes when they have POD.

Laminoplasty is known as one of the most commonly performed surgical procedures for DCM. Thus, this study is aimed to investigate whether the incidence of delirium is higher after laminoplasty than ACDF, which is known as the most common surgical method for the cervical spine, evaluated for comparison with the results of the study. Besides, an analysis of the risk factors was performed to investigate whether the incidence of delirium after cervical spine surgery is affected by a specific disease or surgical method.

\section{Material And Method}

Study population

This study included 148 patients aged 60 years or older who underwent surgery ACDF or laminoplasty surgeries for DCM or myeloradiculopathy at our clinic from 2008 to 2015. Of these 148 patients who underwent surgery, seventy-eight patients underwent laminoplasty, and 70 patients underwent ACDF. In the patients group who underwent laminoplasty, all of them had symptoms and signs associated with myelopathy, and the causes of myelopathy were OPLL $(n=62)$, cervical spinal stenosis $(n=10)$, and herniated nucleus pulposus (HNP) $(n=6)$ (Table 1). Among patients who underwent ACDF, 57 patients had symptoms and signs associated with myeloradiculopathy. The causes of them were HNP $(n=48)$, spinal stenosis $(n=3)$, HNP with spinal stenosis $(n=3)$, and HNP with OPLL $(n=3) .13$ patients who underwent ACDF had only myelopathy and were diagnosed with HNP $(n=6)$, spinal stenosis $(n=2)$, HNP with spinal stenosis $(n=2)$, and HNP with OPLL $(n=3)$ (Table 1$)$.

Table 1. The etiologies of myelopathy. 


\begin{tabular}{|lll|}
\hline & Laminoplasty(N=78) & ACDF (N=70) \\
\hline Myelopathy & & \\
\hline OPLL & 62 & 6 \\
\hline HNP & 6 & 3 \\
\hline HNP with OPLL & 2 \\
\hline HNP with Cervical spinal stenosis & 2 \\
\hline Cervical spinal stenosis & 10 & \\
\hline Myeloradiculopathy & 48 \\
\hline HNP & 3 \\
\hline HNP with OPLL & 3 \\
\hline HNP with Cervical spinal stenosis & 3 \\
\hline Cervical spinal stenosis & \\
\hline
\end{tabular}

OPLL, Ossification of the posterior longitudinal ligament; HNP, Herniated nucleus pulposus

An inclusion criterion was patients who underwent laminoplasty or ACDF aged 60 years or older due to DCM at our institution. Exclusion criteria were patients who have had previous cervical spine surgery, patients who were received a combination of surgery with laminoplasty and ACDF, patients who underwent laminoplasty or ACDF due to myelopathy caused by trauma, tumors and congenital stenosis, pateints who received laminoplasty or ACDF under age 60 years old, and extension of surgical levels to $\mathrm{C} 2$ or $\mathrm{T} 1$. The criteria for ACDF were soft disc herniations, instability of a degenerative nature, concomitant severe axial neck pain, and cervical kyphosis. ACF (Anterior Corpectomy and Fusion) is an alternative to multilevel ACDF, but ACF was not included in our study because of small number of cases. The criteria of laminoplasty were multilevel cervical stensosis, patients with advanced multilevel spondylosis, OPLL, and patients with at least 10 degree of cervical lordosis to allow posterior shift or the spinal cord for indirect compression.

The mean age was 66.7 years (56.5-76.9) in the laminoplasty group and 67.2 years (60.9-73.4) in the ACDF group, with no statistical difference between these two groups $(p=0.747)$. The male to female ratio was 60:18 in the laminoplasty group and 36:34 in the ACDF group, with a statistically significant difference in the Chi-square test $(p=0.02)$. The extent of surgery was 3.13 levels $(2.87-3.39)$ in the laminoplasty group and 1.93 levels (1.74-2.12) in the ADCF group on average, showing a statistically significant difference $(p<0.01)$. The preoperative modified Japanese Orthopaedic Association (mJOA) score for laminoplasty patients was 12.43/18 (8.82-16.04) and that for ACDF patients was 15.16/18 (12.90-17.42), which also showed a statistically significant difference $(p<0.01)$. The postoperative mJOA 
score for laminoplasty patietnts was 14.04/18 (10.51-17.57) and that for ACDF patients was 16.24 (13.93-18.55), showed a statistical difference $(p<0.01)$ (Table 2$)$.

Table 2. Demographics of participants.

\begin{tabular}{|c|c|c|c|c|c|c|}
\hline \multicolumn{7}{|c|}{ Patient who underwent laminoplasty and $\operatorname{ACDF}(n=148)$} \\
\hline \multirow[t]{2}{*}{ Group } & \multicolumn{2}{|c|}{ Laminoplasty $(\mathrm{N}=78)$} & \multicolumn{2}{|c|}{$\mathrm{ACDF}(\mathrm{N}=70)$} & \multirow[t]{2}{*}{$95 \% \mathrm{Cl}$} & \multirow{2}{*}{ palue } \\
\hline & Mean & $\begin{array}{l}\text { Standard } \\
\text { deviation }\end{array}$ & Mean & $\begin{array}{l}\text { Standard } \\
\text { deviation }\end{array}$ & & \\
\hline Age & 66.74 & 10.22 & 67.2 & 6.245 & $\begin{array}{l}-3.246 \sim \\
2.334\end{array}$ & 0.747 \\
\hline Sex ratio(M:F) & $60: 18$ & & $36: 34$ & & & $0.02 *$ \\
\hline Op level & 3.13 & 0.851 & 1.93 & 0.804 & $\begin{array}{l}0.853 \sim \\
1.393\end{array}$ & $<0.01 *$ \\
\hline $\begin{array}{l}\text { mJOA score (Pre- } \\
\text { op.) }\end{array}$ & 12.43 & 3.606 & 15.16 & 2.256 & $\begin{array}{l}-3.726 \sim \\
1.735\end{array}$ & $<0.01 *$ \\
\hline $\begin{array}{l}\text { mJOA score (Post- } \\
\text { op.) }\end{array}$ & 14.04 & 3.528 & 16.24 & 2.312 & $\begin{array}{l}-3.189 \sim \\
1.216\end{array}$ & $<0.01^{*}$ \\
\hline
\end{tabular}

ACDF, Anterior Cervical Discectomy and Fusion; Cl, confidence interval ; mJOA, modified Japanese Orthopedic Association

Chi-square test for sex ratio, Independent t test for age, op level, mJOA score

*Statistical significance $(p<0.05)$.

\section{Surgical technique}

\section{Laminoplasty surgical technique}

A French-door type midline splitting double-door laminoplasty was applied to all cases. The involved spinous processes were split in the midline with a Tomita saw(T-saw; Medtronics, Memphis, TN, USA, $0.54 \mathrm{~mm}$ ) after a posterior incision was made along the nuchal ligament. Then, spinal canal enlargement was made by opening the split laminae bilaterally with a spreader and placing an allo-bone graft (Laminar Spacer-K; CG Bio, Seoul, Korea) after bilateral hinges were made with a burr at the transitional area between the facet joint and laminae. (Fig. 1).

\section{ACDF Surgical technique}

A transverse skin incision was made on the right side of the neck. After approaching to the involved cervical vertebrae, discectomy was performed and allogenic cortical bone graft (Allospacer; CG Bio, Seoul, 
Korea) was applied at each disc space to the appropriate depth. An anterior cervical plate (Atlantis; Medtronics, Memphis, USA) system was then placed over the site of the bone graft (Fig. 2).

\section{Method}

Diagnosis of post-operative delirium (POD)

Based on the medical records of patients receiving replies after consulting a specialist in the neurology or psychiatry department and the medical records that evaluated the patient's condition after surgery, POD were analyzed retrospectively, and the diagnosis of POD was made in the following way.

The surgeons who performed the surgeries and two attending physicians evaluated the cognitive status of patients every day during the period after the surgery until discharge. The evaluation included disturbance in attention (reduced ability to direct, focus, sustain, and shift attention) and disturbance in awareness (reduced orientation to the environment). Based on the Diagnostic and Statistical Manual of Mental Disorders-V criteria (Table 3), delirium was determined: disturbulance occurrence within a few hours or days after the surgery, a change in baseline attention and awareness, and a fluctuation in severity was assessed by asking patients about orientation such as time, place and person and evaluating whether hallucinations or other psychiatric symptoms occurred every eight hours after surgery. 20,21 Besides, patients who did not associate with a pre-existing established neurocognitive disorder were diagnosed with having delirium and underwent consultation with a neurologist or psychiatrist. ${ }^{2,22-24}$

Since the surgeons who performed the surgeries and two attending physicians evaluated the cognitive status of patients were not a neurologists or psychiatrists, authors tried to reduce the error of diagnosis by consulting in a specialized field, and thus seek advice from neurologists or psychiatrists who are experts in the field for a more accurate assessment. The neurologist or psychiatrist reevaluated suspected patients who received consultations and ultimately diagnosed them with delirium. All of the patients described in the paper included only those identified and confirmed as delirium through neurology or psychiatric consultation. The delirium diagnosis was finally determined based on the confusion assessment method (CAM) (Table 3).

Table 3. The Criteria for Diagnosis of postoperative delirium.

\section{Criteria for Diagnosis}

The presence of delirium requires all the criteria to be met:

DSM-5 (Diagnostic and Statistical Manual of Mental disorders, $5^{\text {th }}$ version)

Disturbance in attention and awareness

Disturbance develops acutely and tends to fluctuate in severity

At least one additional disturbance in cognition 
Disturbances are not better explained by a preexisting dementia

Disturbances do not occur in the context of a severely reduced level of arousal or coma

Evidence of an underlying organic cause or causes

CAM (Confusion Assessment Method)

The presence of delirium requires features 1 and 2 and either 3 or 4 :

Acute change in mental status with a fluctuating course (feature 1)

Inattention (feature 2)

Disorganized thinking (feature 3)

Altered level of consciousness (feature 4)

\section{Anesthesia and drug regimen}

All patients underwent cervical spine surgery under general anesthesia, and anesthesia was induced and maintained according to our clinic's standard regimen. Anesthesia was induced with IV propofol and maintained with sevoflurane and remifentanil $(0.25-0.5 \mu \mathrm{g} / \mathrm{kg}$ per $\mathrm{min})$. At the end of the surgery, neostigmine $(0.05 \mathrm{mg} / \mathrm{kg})$ and glycopyrrolate $(0.01 \mathrm{mg} / \mathrm{kg})$ were used to recover from neuromuscular block during extubations. Neostigmine is used to reverse the effect of non-depolarizing muscle relaxants at the end of operation, and glycopyrrolate, which is a medication of the muscarinic anticholinergic group for reducing salivary, tracheobrochial and pharyngeal secretions. They were used once in the process of removing the tube.

\section{Risk factor}

The following known risk factors were measured for each group: age, gender, height, baseline comorbidities (cardiovascular, pulmonary, endocrinal, diabetic, renal and neurologic diseases, Parkinson's disease, liver disease, hypertension, cerebrovascular disease, and dementia), osteoporosis, bone mineral density (BMD) score, hemoglobin $(\mathrm{Hb})$ and hematocrit $(\mathrm{Hct})$, sodium, chloride, potassium, hypotension $(\mathrm{SBP}<80 \mathrm{mmHg}$ ) immediately after the surgery or during surgery, weight, body mass index (BMI), smoking status, American Society of Anaesthesiology (ASA) physical status classification $>2$, amount of blood loss, blood transfusion, transfusion volume, postoperative complications, drugs used to control pain such as opioids, non-steroidal anti-inflammatory drugs(NSAIDs), acetaminophens, and other pain killers, duration of hospitalization, days to wait for hospitalization from admission to surgery, operation time, ICU care, and duration of ICU care. ${ }^{4,5,7,13,14,25,26}$, 
The cross-tabulation analysis was performed by using Pearson's chi-square test for the incidence of POD after laminoplasty and ACDF. In analyzing the risk factors, Pearson's chi-square test (parametric test), Fisher's exact test (nonparametric test), and cross-tabulation analysis were conducted for categorical variables such as transfusion, complication, and occurrence of delirium. An independent T-test (parametric test) and Mann-Whitney $U$ test (nonparametric test) were performed for the remaining continuous variables. Then, a multivariate logistic regression test was conducted on factors with $p$ values lower than 0.05 in subsequent univariate analysis. The IBM SPSS program was used for statistical analysis in this study (IBM SPSS Statistics for Windows version 22.0.0.1 (IBM Corp., 2013, Armonk, NY, USA)). Statistical significance was set at a significance probability ( $p$-value) $<0.05$.

\section{Ethics}

This retrospective study was approved with informed consent waiver by the Institutional Review Board of Hallym University Sacred Heart Hospital (Ref. No. 720127) and performed in accordance with the guidelines and regulations

\section{Results}

Incidence of POD

Delirium occurred in 24 patients out of 148 patients with DCM aged 60 years or older who underwent laminoplasty or ACDF. The incidence of POD was $16.2 \%$. Among those 148 patients who underwent surgery, 78 patients received laminoplasty, and $19(24 \%)$ of them experienced delirium. Seventy patients underwent ACDF and five (7.1\%) of them experienced delirium. The odds ratio (OR) for POD in the laminoplasty and ACDF groups was 4.18 (95\% confidence interval $(\mathrm{Cl})=1.47 \sim 11.9)$. The occurrence of POD in the laminoplasty group was 4.18 times higher than that in the ACDF group, which was statistically significant $(p=0.05)$ (Table 4).

Table 4. Incidence and Odds ratio of postoperative delirium in patients with DCM.

\begin{tabular}{|lllll|}
\hline \multicolumn{4}{|c|}{ Patient who underwent laminoplasty and ACDF(n=148) } & \\
\hline & Laminoplasty & ACDF & Odds ratio(95\% Cl) & P-value \\
\hline Delirium + & $19 / 78(24.3 \%)$ & $5 / 70(7.1 \%)$ & $4.18(1.47 \sim 11.9)$ & $0.050 *$ \\
\hline Delirium - & $59 / 78(75.7 \%)$ & $65 / 70(92.9 \%)$ & & \\
\hline Total & 78 & 70 & & \\
\hline
\end{tabular}

Chi-square test for post-operative delirium; $\mathrm{Cl}$, confidence interval; * Statistical significance $(\mathrm{p}<0.05)$. 
Among 148 patients with DCM aged 60 years or older who underwent surgery, the factors that found to be statistically significant were age, neurologic disease, dementia, Parkinson's disease, stroke history, sodium level, potassium level, and ASA classification $>2$, transfusion volume, blood transfusion, and ICU care. (Age: $P<0.001$, neurologic disease: $P=0.009$, dementia: $P=0.001$, Parkinson's disease: $P=0.001$, Stroke history: $P=0.022$, sodium: $P=0.012$, potassium: $P=0.048$, $A S A>2: P=0.002$, transfusion volume: $P=0.013$, blood transfusion: $P=0.001$, ICU care: $P=0.003)($ Table 5)

Table 5. Analysis of risk factors for postoperative delirium in patients with DCM. 


\begin{tabular}{|c|c|c|c|}
\hline & $\begin{array}{l}\text { Post Operative } \\
\text { Delirium - } \\
(\mathrm{N}=124)\end{array}$ & $\begin{array}{l}\text { Post Operative } \\
\text { Delirium + } \\
(\mathrm{N}=24)\end{array}$ & P Value \\
\hline Gender & & & 0.503 \\
\hline Male & 79(63.7\%) & 17(70.8\%) & \\
\hline Female & $45(36.2 \%)$ & $7(29.1 \%)$ & \\
\hline Age (years) & $73.2 \pm 10.2$ & $65.7 \pm 7.66$ & $0.000 *$ \\
\hline Smoking & $22(17.7 \%)$ & $5(20.8 \%)$ & 0.72 \\
\hline Cardiovascular disease & $41(33.0 \%)$ & $6(25 \%)$ & 0.437 \\
\hline Pulmonary disease & $9(7.25 \%)$ & $1(4.16 \%)$ & 0.581 \\
\hline Endocrinal disease & $30(24.1 \%)$ & $8(33.3 \%)$ & 0.348 \\
\hline DM & $27(21.7 \%)$ & $7(29.1 \%)$ & 0.431 \\
\hline Renal disease & $4(3.22 \%)$ & $2(8.33 \%)$ & 0.246 \\
\hline Neurologic disease & $12(9.67 \%)$ & $7(29.1 \%)$ & $0.009 *$ \\
\hline Dementia & $0(0 \%)$ & $2(8.33 \%)$ & $0.001^{\star}$ \\
\hline Parkinson's disease & $1(0.80 \%)$ & $3(12.5 \%)$ & $0.001^{*}$ \\
\hline Liver disease & $5(4.03 \%)$ & $1(4.16 \%)$ & 0.976 \\
\hline HTN & $52(41.9 \%)$ & $13(54.1 \%)$ & 0.269 \\
\hline Stroke & $3(2.41 \%)$ & $3(12.5 \%)$ & $0.022^{*}$ \\
\hline MDD & $6(4.83 \%)$ & $0(0 \%)$ & 0.271 \\
\hline Osteoporosis & $5(4.03 \%)$ & $1(4.16 \%)$ & 0.976 \\
\hline BMD score & $-1.1 \pm 1.74$ & $-0.7 \pm 1.56$ & 0.427 \\
\hline $\mathrm{Hb}$ & $13.0 \pm 1.97$ & $13.7 \pm 1.47$ & 0.35 \\
\hline Hct & $36.5 \pm 3.18$ & $37.7 \pm 3.91$ & 0.201 \\
\hline Sodium & $138 . \pm 4.78$ & $140 . \pm 3.62$ & $0.012^{\star}$ \\
\hline Chloride & $102 . \pm 3.80$ & $103 . \pm 3.15$ & 0.077 \\
\hline Potassium & $4.47 \pm 0.54$ & $4.28 \pm 0.41$ & $0.048^{*}$ \\
\hline Intra-operative hypotension & $5(4.03 \%)$ & $1(4.16 \%)$ & 0.976 \\
\hline Height (cm) & $161.97 \pm 8.50$ & $160.90 \pm 8.22$ & 0.804 \\
\hline
\end{tabular}




\begin{tabular}{|llll|}
\hline Weight $(\mathrm{Kg})$ & $62.7 \pm 13.0$ & $65.1 \pm 11.1$ & 0.35 \\
\hline BMI & $24.1 \pm 3.99$ & $24.8 \pm 3.59$ & 0.42 \\
\hline ASA class $>2$ & $37(29.8 \%)$ & $15(62.5 \%)$ & $0.002^{\star}$ \\
\hline Intra-operative blood loss $(\mathrm{mL})$ & $787.5 \pm 550.3$ & $652.3 \pm 368.4$ & 0.134 \\
\hline Blood Transfusion & $22(17.7 \%)$ & $12(50 \%)$ & $0.001^{\star}$ \\
\hline Transfusion volume $(\mathrm{mL})$ & $363.3 \pm 423.9$ & $144.5 \pm 383.5$ & $0.013^{\star}$ \\
\hline Post-operative Opioid use & $17(13.7 \%)$ & $4(16.6 \%)$ & 0.704 \\
\hline Post-operative NSAIDs use & $77(62.0 \%)$ & $16(66.6 \%)$ & 0.672 \\
\hline Post-operative Acetaminophen use & $50(40.3 \%)$ & $13(54.1 \%)$ & 0.209 \\
\hline Post-operative other pain killer use & $58(46.7 \%)$ & $8(33.3 \%)$ & 0.225 \\
\hline Post-operative complications & $19(15.3 \%)$ & $7(29.1 \%)$ & 0.103 \\
\hline Duration of admission (days) & $18.3 \pm 11.2$ & $19.9 \pm 11.1$ & 0.58 \\
\hline Duration of pre-operative period (days) & $3.9 \pm 7.2$ & $2.3 \pm 1.7$ & 0.337 \\
\hline Operation time(mins) & $245.75 \pm 89.98$ & $240.83 \pm 81.79$ & 0.804 \\
\hline ICU care & $19(15.3 \%)$ & $10(41.6 \%)$ & $0.003^{\star}$ \\
\hline Duration of ICU care period (days) & $0.87 \pm 2.04$ & $0.67 \pm 3.71$ & 0.801 \\
\hline
\end{tabular}

DM, Diabetes Mellitus; HTN, Hypertension; MDD, Manic Depressive Disorders; BMD, Bone Mineral Density; ASA, American Society of Anestheologist, NSAIDs, Non-Steroidal Anti-Inflammatory Drugs * Statistical significance $(p<0.05)$. The number after \pm means standard deviation.

Multivariate logistic regression analysis was performed on the above factors. Only age (OR=-0.09, 95\% $\mathrm{Cl}=0.834-0.990, \mathrm{p}=0.029)$, and sodium level $(\mathrm{OR}=-0.153,95 \% \mathrm{Cl}=1.009-1.347, \mathrm{p}=0.037)$ were statistically significant for the development of POD. (Table 6)

Table 6. Multivariate analysis of risk factors of postoperative delirium in patients with DCM. 


\begin{tabular}{|llll|}
\hline Total Patients $(\mathrm{n}=148)$ & \multicolumn{3}{l|}{ Multivariate analysis } \\
\cline { 2 - 4 } & Odds Ratio & $95 \% \mathrm{Cl}$ & $\mathrm{p}$-value \\
\hline Age & -0.09 & $0.834 \sim 0.990$ & $0.029 *$ \\
\hline Neurologic disease & 20.12 & & 0.998 \\
\hline Dementia & -0.89 & & 1 \\
\hline Parkinson's disease & 19.43 & & 0.999 \\
\hline Stroke & -20.6 & & 0.998 \\
\hline Sodium & 0.153 & $1.009 \sim 1.347$ & $0.037 *$ \\
\hline Potassium & -0.44 & $0.145 \sim 2.794$ & 0.552 \\
\hline ASA class $>2$ & -0.23 & $0.191 \sim 3.269$ & 0.746 \\
\hline Transfusion volume & -0.001 & $0.997 \sim 1.001$ & 0.595 \\
\hline Blood Transfusion & 0.285 & $0.131 \sim 13.46$ & 0.809 \\
\hline ICU & -0.89 & $0.092 \sim 1.791$ & 0.235 \\
\hline
\end{tabular}

$\mathrm{Cl}$, confidence interval; ASA, American Society of Anesthesiologist; ICU, Intensive care unit *Statistical significance $(p<0.05)$.

On the other hand, in the patient group who underwent laminoplasty, the factors that found to be statistically significant were the patient's age $(p=0.008)$, Parkinson's disease $(p=0.012)$, sodium level ( $p$ $=0.023)$, potassium level $(0.047)$, and ICU care $(p=0.033)$. Other factors were not statistically significant (Table 7).

Multivariate logistic regression analysis was performed on the above factors. Only age (OR=1.056, $95 \%$ $\mathrm{Cl}=1.004 \sim 1.124, \mathrm{p}=0.04)$, and potassium level (OR 3.217, $\mathrm{Cl}=1.456-11.174, \mathrm{p}=0.04)$ were statistically significant for the development of POD in the patients who underwent laminoplasty (Table 8). This was not analyzed in ACDF due to the low $n(=5)$.

Table 7. Analysis of risk factors for postoperative delirium following laminoplasty. 


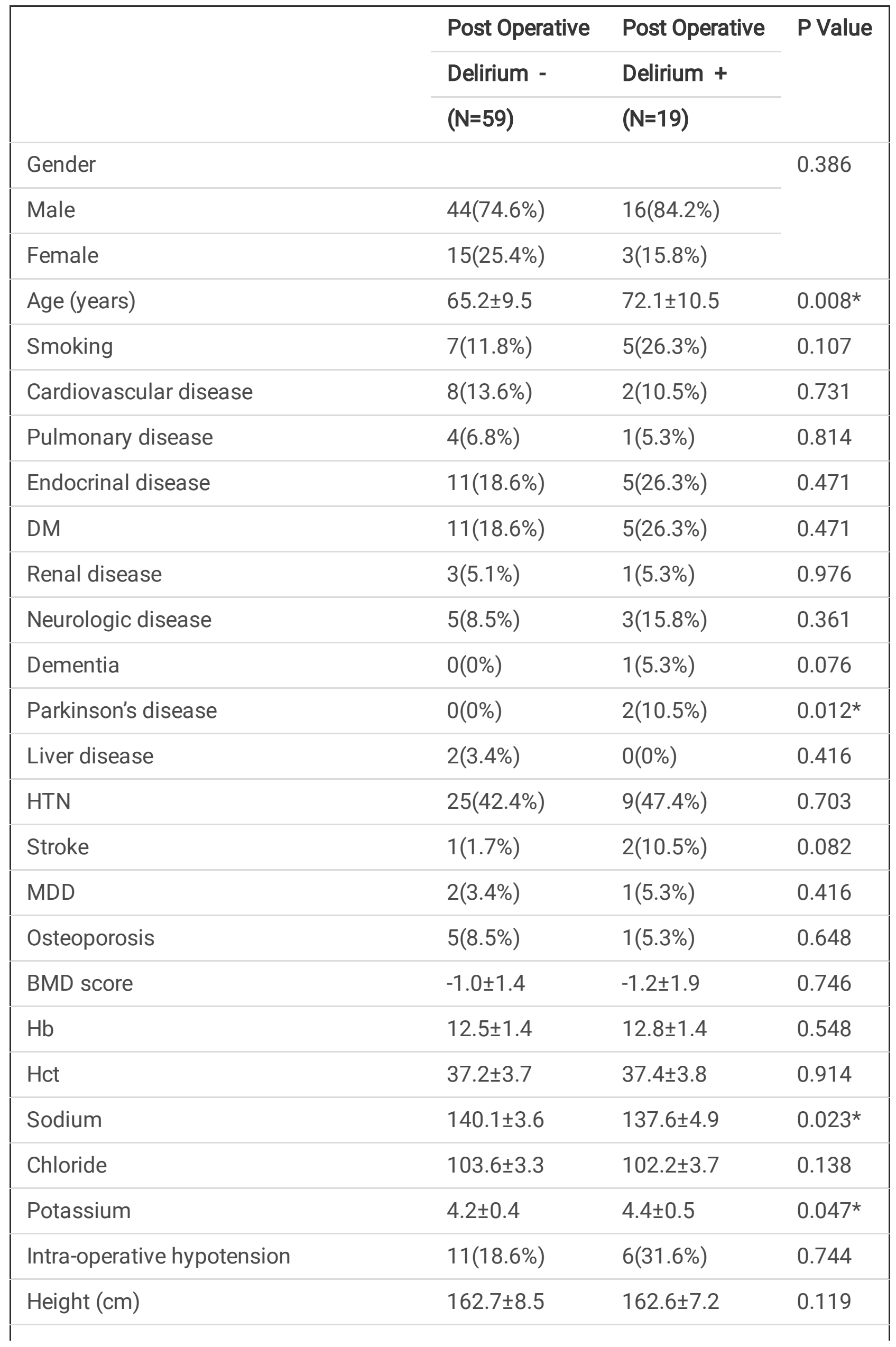




\begin{tabular}{|llll|}
\hline Weight $(\mathrm{Kg})$ & $65.8 \pm 9.8$ & $62.3 \pm 11.0$ & 0.16 \\
\hline BSI & $24.8 \pm 2.5$ & $23.5 \pm 3.1$ & 0.201 \\
\hline Intra-operative blood loss $(\mathrm{mL})$ & $24(40.7 \%)$ & $11(57.9 \%)$ & 0.189 \\
\hline Blood Transfusion & $683.9 \pm 297.9$ & $828.6 \pm 342.9$ & 0.558 \\
\hline Transfusion volume $(\mathrm{mL})$ & $13(22.0 \%)$ & $8(42.1 \%)$ & 0.086 \\
\hline Post-operative Opioid use & $180.4 \pm 439.5$ & $290.5 \pm 395.7$ & 0.335 \\
\hline Post-operative NSAIDs use & $12(20.3 \%)$ & $3(15.7 \%)$ & 0.662 \\
\hline Post-operative Acetaminophen use & $38(64.4 \%)$ & $13(68.4 \%)$ & 0.749 \\
\hline Post-operative other pain killer use & $28(47.4 \%)$ & $6(31.6 \%)$ & 0.225 \\
\hline Post-operative complications & $4(6.8 \%)$ & $2(10.5 \%)$ & 0.311 \\
\hline Duration of admission (days) & $18.3 \pm 11.2$ & $19.9 \pm 11.1$ & 0.58 \\
\hline Duration of pre-operative period (days) & $3.9 \pm 7.2$ & $2.3 \pm 1.7$ & 0.337 \\
\hline Operation time (mins) & $253.2 \pm 57.9$ & $253.2 \pm 62.2$ & 0.715 \\
\hline Duration of post-operative period (days) & $14.3 \pm 10.7$ & $17.6 \pm 11.2$ & 0.58 \\
\hline ICU care & $13(22.0 \%)$ & $9(47.4 \%)$ & $0.033 *$ \\
\hline Duration of ICU care period (days) & $1.2 \pm 5.3$ & $1.0 \pm 2.2$ & 0.918 \\
\hline
\end{tabular}

DM, Diabetes Mellitus; HTN, Hypertension; MDD, Manic Depressive Disorders; BMD, Bone Mineral Density; ASA, American Society of Anestheologist, NSAIDs, Non-Steroidal Anti-Inflammatory Drugs * Statistical significance $(p<0.05)$. The number after \pm means standard deviation.

Table 8. Multivariate analysis of risk factors of postoperative delirium following laminoplasty.

\begin{tabular}{|llll|}
\hline Laminoplasty & \multicolumn{3}{l|}{ Multivariate analysis } \\
\cline { 2 - 4 } & Odds Ratio & $95 \% \mathrm{Cl}$ & P-value \\
\hline Age & 1.056 & $1.004 \sim 1.124$ & $0.04^{*}$ \\
\hline Parkinson disease & 154 & $0.000 \sim 154$ & 0.99 \\
\hline ICU care & 2.457 & $0.658 \sim 7.037$ & 0.17 \\
\hline Sodium & 0.879 & $0.762 \sim 1.013$ & 0.75 \\
\hline Potassium & 3.217 & $1.456 \sim 11.174$ & $0.04^{\star}$ \\
\hline
\end{tabular}


$\mathrm{Cl}$, confidence interval; ICU, Intensive care unit *Statistical significance $(\mathrm{p}<0.05)$.

\section{Discussion}

Several studies on POD have been conducted on patients that underwent spinal surgery, investigating the incidence and risk factors. According to the literature, the incidence of the delirium after spinal surgery is reported at $11-61 \% .{ }^{12-14,26}$ Various risk factors have also been reported. ${ }^{12,23,26,27}$ According to a retrospective study, which analyzed data from more than 500,000 patients with various degenerative diseases of the lumbar spine including herniated lumbar discs, spondylolisthesis, and lumbar spinal stenosis, the risk factors of POD were identified as age ( $\geq 65 \mathrm{yr}$ ), gender (female gender), alcohol/drug abuse, depression, psychotic disorders, and neurological disorders. ${ }^{13}$

However, previous studies on spinal surgery have covered on a wide range of spinal disorders including HNP (Herniated Nucleus Pulposus), spinal stenosis, myelopathy, tumors, and trauma across a variety of regions treated with various surgical methods, they did not characterize the POD associated with certain specific disorders. ${ }^{23,27,28}$ Furthermore, although not many, some of meta-analysis were conducted by extracting data from other studies, the methods for determining POD were heterogeneous. ${ }^{12}$ Like this, previous studies aimed at all kinds of different subjects providing no meaningful information in analyzing the difference in the incidence and risk factors of POD resulting from the severity and characteristics of a particular disease.

\section{Incidence of $P O D$}

In the study, delirium occurred in $24(16.2 \%)$ out of 148 elderly patients aged 60 years or older who underwent cervical spine surgery, similar to the results of previous studies, which reported an incidence of $11 \%$ - 61\%. (Table 4) $)^{12-14,26}$

However, analysis on the prevalence of delirium depending on the surgical method revealed that 19 (24\%) out of 78 patients who underwent laminoplasty experienced delirium, while 59 patients did not $(76 \%)$. On the other hand, only five (7.1\%) out of 70 patients who underwent ACDF experienced delirium, while 65 patients did not (92.9\%). The OR for POD in the laminoplasty and ACDF groups was $4.18(95 \% \mathrm{Cl}=1.47$ 11.9). The occurrence of POD in the laminoplasty group was 4.18 times higher than that in the ACDF group, which was statistically significant $(p=0.05)$ (Table 4). This result shows that there is a difference in the development of POD depending on the surgical method even for the same disease. This finding deviates substantially from those of previous similar studies. Previous studies analyzed various factors that affect the incidence of delirium for all spinal surgeries without considering the surgical regions or methods, the characteristics of subjects, or the prevalence or factors that affect the incidence of delirium in one category for all surgical methods. These studies disregarded the characteristics of specific surgical methods, which could be chosen depending on the severity of a patient's disease, despite surgery on the same area (e.g., cervical spine). As a result, the studies overlooked the characteristics present in the incidence of delirium. This approach could make medical professionals overly cautious about the 
incidence of delirium for surgeries with a low frequency of delirium, while making them erroneously comfortable with surgeries with a high risk of delirium incidence. Thus, it should be noted that POD after spinal surgery can vary depending on the type and selection of surgical methods. This is particularly related to the fact that spinal surgery, unlike surgery in any other parts, has been performed through various methods depending on the surgical levels and the characteristics of the disease, despite surgery for a similar disease in the same area. For example, in cases of avascular necrosis of the femoral head at the hip joint, total knee replacement arthroplasty is regarded as the most commonly or solely selected surgical method and other surgical methods are rarely performed. On the other hand, as for spinal surgery, anterior or posterior surgical approaches and the various surgical methods are selected by surgeons depending on the degree and severity of disease, even operated on a similar disease in the same area.

\section{Risk Factors of the POD}

According to this study, the risk factors that affect the incidence of POD following cervical spine surgery were age, neurologic disease, dementia, Parkinson's disease, stroke history, hemoglobin, sodium level, potassium level, and ASA classification > 2, transfusion volume, blood transfusion, and ICU care (Table 5). Among these factors, only age $(\mathrm{OR}=-0.09,95 \% \mathrm{Cl}=0.834 \sim 0.990, \mathrm{p}=0.029)$, and sodium level $(\mathrm{OR}=-0.153$, $95 \% \mathrm{Cl}=1.009 \sim 1.347, \mathrm{p}=0.037$ ) were statistically significant for the development of POD in multiple regression analysis (Table 6). When we looked at the factors involved in the occurrence of the POD for laminoplasty surgery, the factors that found to be statistically significant were patient's age $(p=0.008)$, Parkinson's disease $(p=0.012)$, sodium level $(p=0.023)$, potassium level $(0.047)$, ICU care $(p=0.033)$ in patients who underwent laminoplasty.(Table 7) A multivariate logistic regression test was conducted on the above factors and revealed that age $(\mathrm{OR}=1.056,95 \% \mathrm{Cl}=1.004 \sim 1.124, \mathrm{p}=0.04)$ and potassium level (OR 3.217, $\mathrm{Cl}=1.456 \sim 11.174, \mathrm{p}=0.04$ ) alone were significant factors in the development of POD (Table 8). Thus, if any patient who receives laminoplasty due to DCM is old or has an electrolyte imbalance (high potassium level), he or she will require more caution due to a higher probability of POD. However, the factors affect ACDF were not analyzed in the study, because the incidence of POD unexpectedly lowers in ACDF $(n=5)$, making it difficult to statistically analyze the associated factors and have a statistical significance with it.

In the study, the authors have tried to investigate factors that are known to affect spinal surgery, and examined the relations between these factors and the development of POD.

Smoking is known as one of the factors that affects the outcome of surgery. ${ }^{29}$ Toxic agents come from cigarette smoke could lead to atherosclerotic and microvascular changes. They gradually build up and remain in the body for a long time even after quitting. These changes are highly linked to cognitive dysfunction, dementia and the risk of developing delirium. ${ }^{9}$ However, In our study, smoking was not found to increase the risk of POD.(Table 5,7) Dementia and delirium are also known to be related to drug toxicity. ${ }^{30}$ Especially, cognitive impairments associated with medications are more common in elderly people, because renal and liver functions are often impaired. Anticholinergic medications are main 
reasons of both acute and chronic cognitive impairment. Psychoactive drugs, antidepressants and anticonvulsants can lead to dementia and delirium. Non-psychoactive drugs such as histamine $\mathrm{H} 2$ receptor antagonists, corticosteroids, NSAIDs (nonsteroidal anti-inflammatory agent), and cardiac medications may cause cognitive dysfunction. Early diagnosis and withdrawal of the offending agent are necessary for the prevention of drug-induced dementia and delirium. However, NSAIDs were not found to be a meaningful factor in our study. (Table 5,7 ) Opioids have a relatively different risk of dementia. ${ }^{31}$ Meperidine is associated with an increased risk of delirium compared with other opioids, whereas tramadol is not. On the other hand, morphine, fentanyl, oxycodone, and codeine are not related to the development of delirium when compared with non- opioid. Hydromorphone or fentanyl has a relatively lower risk of delirium as compared with other opioids. Meperidine has a higher perioperative risk for delirium.

In our study, pain killers and opioids did not affect the development of POD, however, drugs mentioned above may affect the POD, so care should be taken to use painkillers after surgery. (Table 5,7$)$ Other factors associated with surgery including blood transfusion, operation, blood loss, operation time, postoperative complications, ICU care, duration of admission etc were not found to be significant.

In addition, patient with existing neurological disorders such as dementia or Parkinson disease have been reported to develop POD, and authors included them as one of the causes of the delirium in the study. A recent systematic review suggested that people with Parkinson disease (PD) may be at increased risk of delirium. ${ }^{11}$

According to a study of Caplan et al, $^{10}$ diagnosing delirium in the presence of pre-existing dementia is difficult and gets harder as either progresses because their symptoms are intertwined. Prolonged delirium becomes permanent cognitive impairment, and severe dementia is often manifested by multiple symptoms that are similar to those of delirium. As the neuroinflammatroy mechanism in delirium is actually mediated by the effect of glucose, and not the inflammatory changes in the brain, they suggest that glucose could be the link between dementia and delirium pathophysiology, given that dementia is characterized by insulin resistance and impairments in glucose metabolism and delirium may be working likewise. As if to support this hypothesis, recent studies of FDG-PET (F-fluorodeoxyglucose positron emission tomography) in delirium reveal a novel and unique pattern of glucose hypometabolism that correlates with neuropsychological functions. Parkinson disease, dementia and neurologic disease were found to be meaningful factor in the occurrence of POD in cervical spine surgery, and Parkinson disease was also a meaningful factor in laminoplasty group in the study (Table 5,7$)$. Therefore, patients with neurological disorders such as Parkinson disease and dementia need to take special care of the occurrence of POD if they operate on cervical spine surgery as well as laminoplasty. Preoperative comorbidities such as liver disease, renal disease, cardiovascular disease, pulmonary disease, hypertension, endocrine disease, DM, BMI, BMD and osteoporosis were also analyzed but they were not found to be meaningful factors associated with POD in the study. There is little research on whether gender affects the occurrence of the POD. According to a study ${ }^{32}$, delirium was more often affected men amongst patients under 65 years old and more frequently affected women in the age group of over 85 
years old. Age was also found to a meaningful factor to develop the POD in our study, however, gender was not found to be a meaningful risk factor of POD (Table 5,7$)$.

Considering the above results from the study, only age was not only a significant factor for the entire cervical spine surgery, but also for laminoplasty. The other factors differed from surgery to surgery. As with other surgeries, special attention should be paid to the occurrence of the POD in older patients.

\section{Reasons for a difference in POD}

Based on our observations, there was a difference in the occurrence of delirium between ACDF and laminoplasty in the study. This might be accepted as an important argument. We potentially suggest the reason why the incidence of POD was higher following laminoplasty than ACDF as follows:

The preoperative mJOA score was $12.43 / 18$ in the laminoplasty group and that of the ACDF group was $15.16 / 18$. The preoperative mJOA score was statistically significantly low in the laminoplasty group $(p<0.001,95 \% \mathrm{Cl}-3.726 \sim 1.735)$ (Table 2), suggesting the preoperative neurological status in the patients who underwent laminoplasty was worse and this status might influence the development of POD.

In addition, the operation levels were 1.93 levels in the ACDF group and 3.13 levels in the laminoplasty group on average, which indicates that the extent of surgery was relatively wider for laminoplasty $(p<0.001,95 \% \mathrm{Cl} 0.853 \sim 1.393)$ (Table 1). Because the surgical area was wider and more extensive in the patient group that underwent laminoplasty, the patients would have considered the operation to be more serious. Furthermore, 74 patients underwent laminoplasty mainly due to the symptoms of myelopathy, characterized by hand clumsiness, gait disturbance, and bowel or bladder dysfunctions, while the other group of patients underwent ACDF mainly due to symptoms of myeloradiculopathy (77\%) accompanied by radiculopathy and mild myelopathy. For these reasons, the authors inferred that the incidence of POD was relatively higher in the laminoplasty group than in the ACDF group, which was commonly performed in cervical spine surgery. Further studies are required to determine whether these reasons actually contributed to the incidence of POD.

\section{Diagnosis and treatment of the $P O D$}

In this study, we tried to diagnose it using the diagnostic criteria and methods currently in practice at the clinical site (DSM-V) ${ }^{33,34}$, and tried to double-check it through consultation with neurology or psychiatry, the specialty of the disease.

According to the guidance of the specialists, various treatments, ranging from drug treatment to supportive therapy, were carried out depending on the degree of the patient's symptoms. The treatment of POD combines drug therapy and supportive treatment for the situation. ${ }^{20,35}$ First of all, it is necessary to secure hemodynamic stability and correct electrolyte imbalance after surgery. If the symptoms are not severe, and in the early stages, it continuously provides information on time, place, and person, and creates an environment where patients can sleep without interruption during bedtime. Fast walking and 
movement can also help treat patients, and it is also necessary to create an environment to help patients recover by using the items needed for each patient.

\section{Limitations}

This study has some limitations as follows. First, this study, as a retrospective study, has a limitation in that the sample size was not sufficiently large because the study was conducted at a single institution. Second, as in this study, as far as authors know, there are very few research methods that objectively evaluate the preoperative cognitive function of patients. All of the patients who participated in this study showed no cognitive dysfunction in the preoperative evaluation according to the criteria of DSM-5 and CAM. However, future studies show that the methods and data of objectively assessing the cognitive status of patients before surgery, along with objectively assessing the cognitive function of patients after surgery, can be one of the key factors in determining the results of the study associated with POD. Third, although we intended to compare and analyze factors affecting the POD according to the method of surgery in DCM, we'd like to emphasize that we can create a bias in the interpretation of the results by not including all factors that may be related to the occurrence of POD. In addition, in the course of analyzing the factors affecting the occurrence of POD between surgical methods, the incidence of POD unexpectedly lowers in $\operatorname{ACDF}(n=5)$, making it difficult to statistically analyze the associated factors and have a statistical meaning with it. We look forward to further research on this. Lastly, this study also did not include other surgical methods for DCM, such as posterior laminectomy and fusion or anterior corpectomy and fusion, due to the small number of cases. As it is not easy to review the characteristics depending on all surgical methods for DCM, this study was focused on laminoplasty, which is regarded as one of the most common surgical procedures in Asia for DCM.

\section{Conclusion}

The incidence and risk factors for POD can vary depending on the surgical method employed in patients with DCM. In addition to the relatively high risk of POD following laminoplasty, it is necessary to understand factors that affect the POD.

\section{Declarations}

\section{Data availability}

All data generated or analyzed during this study will be made available by the corresponding author on reasonable request.

\section{Acknowledgments}

SK was supported by Hallym University Research Fund (HRF-01-2010-13) and National Research Foundation of Korea (2015R1D1A1A01061562). 


\section{Author contributions}

S.W.K was responsible for the whole project and supervised the study. S.W.K designed the concept and described the manuscript. S.H.L collected the data. J.H.L and H.M.L analyzed the data and drafted the article. M.U.L and Y.E.K edited the article for important intellectual content. All authors read and approved the final version of the manuscript.

\section{Competing interests}

The authors declare no competing interests.

\section{References}

1 Ely, E. W. et al. Evaluation of delirium in critically ill patients: validation of the Confusion Assessment Method for the Intensive Care Unit (CAM-ICU). Critical care medicine29, 1370-1379, doi:10.1097/00003246-200107000-00012 (2001).

2 Inouye, S. K. et al. Clarifying confusion: the confusion assessment method. A new method for detection of delirium. Annals of internal medicine113, 941-948, doi:10.7326/0003-4819-113-12-941 (1990).

3 Kalakoti, P. et al. Inpatient Outcomes and Postoperative Complications After Primary Versus Revision Lumbar Spinal Fusion Surgeries for Degenerative Lumbar Disc Disease: A National (Nationwide) Inpatient Sample Analysis, 2002-2011. World neurosurgery85, 114-124, doi:10.1016/j.wneu.2015.08.020 (2016).

4 Takeuchi, M. et al. Incidence and risk factors of postoperative delirium in patients with esophageal cancer. Annals of surgical oncology19, 3963-3970, doi:10.1245/s10434-012-2432-1 (2012).

5 Brauer, C., Morrison, R. S., Silberzweig, S. B. \& Siu, A. L. The cause of delirium in patients with hip fracture. Archives of internal medicine160, 1856-1860, doi:10.1001/archinte.160.12.1856 (2000).

6 Marcantonio, E. R. Postoperative delirium: a 76-year-old woman with delirium following surgery. Jama308, 73-81, doi:10.1001/jama.2012.6857 (2012).

7 Bin Abd Razak, H. R. \& Yung, W. Y. Postoperative Delirium in Patients Undergoing Total Joint Arthroplasty: A Systematic Review. The Journal of arthroplasty30, 1414-1417, doi:10.1016/j.arth.2015.03.012 (2015).

8 Koster, S., Hensens, A. G., Schuurmans, M. J. \& van der Palen, J. Risk factors of delirium after cardiac surgery: a systematic review. European journal of cardiovascular nursing : journal of the Working Group on Cardiovascular Nursing of the European Society of Cardiology10, 197-204, doi:10.1016/j.ejcnurse.2010.09.001 (2011). 
9 Raats, J. W., Steunenberg, S. L., de Lange, D. C. \& van der Laan, L. Risk factors of post-operative delirium after elective vascular surgery in the elderly: A systematic review. International journal of surgery (London, England)35, 1-6, doi:10.1016/j.ijsu.2016.09.001 (2016).

10 Caplan, G. A. Delirium Superimposed Upon Dementia. Journal of the American Medical Directors Association20, 1382-1383, doi:10.1016/j.jamda.2019.09.019 (2019).

11 Lawson, R. A., Richardson, S. J., Yarnall, A. J., Burn, D. J. \& Allan, L. M. Identifying delirium in Parkinson disease: A pilot study. Int J Geriatr Psychiatry, doi:10.1002/gps.5270 (2020).

12 Zhu, C. et al. Risk factors for postoperative delirium after spinal surgery: a systematic review and meta-analysis. Aging Clin Exp Res, doi:10.1007/s40520-019-01319-y (2019).

13 Fineberg, S. J. et al. Incidence and risk factors for postoperative delirium after lumbar spine surgery. Spine38, 1790-1796, doi:10.1097/BRS.0b013e3182a0d507 (2013).

14 Ushida, T. et al. Incidence and risk factors of postoperative delirium in cervical spine surgery. Spine34, 2500-2504, doi:10.1097/BRS.0b013e3181b321e6 (2009).

15 Tetreault, L. et al. Degenerative Cervical Myelopathy: A Spectrum of Related Disorders Affecting the Aging Spine. Neurosurgery77, S51-S67, doi:10.1227/Neu.0000000000000951 (2015).

16 Toledano, M. \& Bartleson, J. D. Cervical spondylotic myelopathy. Neurologic clinics31, 287-305, doi:10.1016/j.ncl.2012.09.003 (2013).

17 Iyer, A., Azad, T. D. \& Tharin, S. Cervical Spondylotic Myelopathy. Clinical spine surgery29, 408-414, doi:10.1097/bsd.0000000000000397 (2016).

18 Fehlings, M. G. et al. Anterior versus posterior surgical approaches to treat cervical spondylotic myelopathy: outcomes of the prospective multicenter AOSpine North America CSM study in 264 patients. Spine (Phila Pa 1976)38, 2247-2252, doi:10.1097/BRS.0000000000000047 (2013).

19 Lawrence, B. D. et al. Anterior versus posterior approach for treatment of cervical spondylotic myelopathy: a systematic review. Spine (Phila Pa 1976)38, S173-182, doi:10.1097/BRS.0b013e3182a7eaaf (2013).

20 Robinson, T. N. et al. Postoperative delirium in the elderly: risk factors and outcomes. Ann Surg249, 173-178, doi:10.1097/SLA.0b013e31818e4776 (2009).

21 Marcantonio, E. R. et al. A clinical prediction rule for delirium after elective noncardiac surgery. JAMA271, 134-139 (1994).

22 Cavallari, M. et al. Longitudinal diffusion changes following postoperative delirium in older people without dementia. Neurology89, 1020-1027, doi:10.1212/WNL.0000000000004329 (2017). 
23 Brown, C. H. t. et al. Delirium After Spine Surgery in Older Adults: Incidence, Risk Factors, and Outcomes. J Am Geriatr Soc64, 2101-2108, doi:10.1111/jgs.14434 (2016).

24 Rudolph, J. L. et al. Delirium is associated with early postoperative cognitive dysfunction. Anaesthesia63, 941-947, doi:10.1111/j.1365-2044.2008.05523.x (2008).

25 Takeuchi, D. et al. Incidence, Clinical Course, and Risk Factors of Amiodarone-Induced Thyroid Dysfunction in Japanese Adults With Congenital Heart Disease. Circ J9, 1828-1834, doi:10.1253/circj.CJ-15-0042 (2015).

26 Morino, T. et al. Risk Factors for Delirium after Spine Surgery: An Age-Matched Analysis. Asian Spine J12, 703-709, doi:10.31616/asj.2018.12.4.703 (2018).

27 Susano, M. J. et al. Retrospective Analysis of Perioperative Variables Associated With Postoperative Delirium and Other Adverse Outcomes in Older Patients After Spine Surgery. J Neurosurg Anesthesio/31, 385-391, doi:10.1097/ANA.0000000000000566 (2019).

28 Elsamadicy, A. A. et al. Association of Intraoperative Blood Transfusions on Postoperative Complications, 30-Day Readmission Rates, and 1-Year Patient-Reported Outcomes. Spine42, 610-615, doi:10.1097/Brs.0000000000001803 (2017).

29 Hessler, J. B. et al. Smoking increases the risk of delirium for older inpatients: a prospective population-based study. Gen Hosp Psychiatry37, 360-364, doi:10.1016/j.genhosppsych.2015.03.009 (2015).

30 Shinohara, Y. Delirium caused by intramuscular administration of biperiden. J Neuropsychiatry Clin Neurosci22, E15, doi:10.1176/appi.neuropsych.22.2.247-i.e1510.1176/jnp.2010.22.2.247.e15 (2010).

31 Swart, L. M., van der Zanden, V., Spies, P. E., de Rooij, S. E. \& van Munster, B. C. The Comparative Risk of Delirium with Different Opioids: A Systematic Review. Drugs Aging34, 437-443, doi:10.1007/s40266017-0455-9 (2017).

32 Serpytis, P. et al. Age- and gender-related peculiarities of patients with delirium in the cardiac intensive care unit. Kardiol Pol75, 1041-1050, doi:10.5603/KP.a2017.0122 (2017).

33 European Delirium, A. \& American Delirium, S. The DSM-5 criteria, level of arousal and delirium diagnosis: inclusiveness is safer. BMC Med12, 141, doi:10.1186/s12916-014-0141-2 (2014).

34 Sepulveda, E. et al. Delirium diagnosis defined by cluster analysis of symptoms versus diagnosis by DSM and ICD criteria: diagnostic accuracy study. BMC Psychiatry 16,167 , doi:10.1186/s12888-016-08786 (2016).

35 Robinson, T. N. \& Eiseman, B. Postoperative delirium in the elderly: diagnosis and management. Clin Interv Aging3, 351-355, doi:10.2147/cia.s2759 (2008). 


\section{Figures}

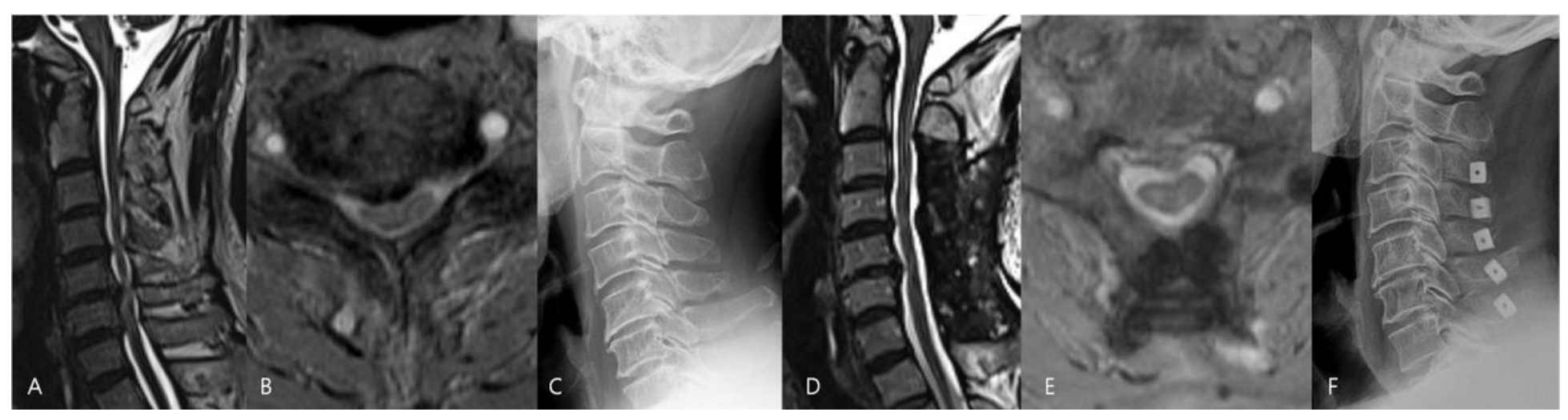

\section{Figure 1}

Pre-operative and post-operative radiographic images of 70-year-old male who underwent laminoplasty; Sagittal (A) and axial (B) images of pre-operative T2-weighted MRI, pre-operative cervical spine lateral Xray (C), sagittal(D) and axial(E) images of post-operative T2-weighted MRI, post-operative cervical spine lateral $x$-ray (F) for 70-year-old male patient who underwent midline splitting double-door laminopalsty (French door type).

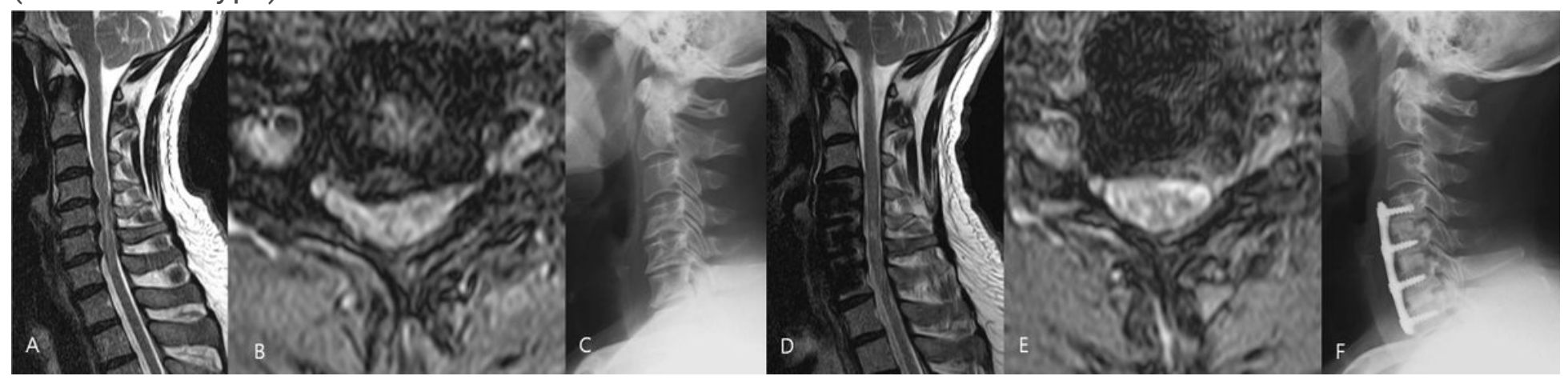

\section{Figure 2}

Pre-operative and post-operative radiographic images of 69-year-old male who underwent ACDF; Sagittal $(A)$ and axial (B) images of pre-operative T2-weighted MRI, pre-operative cervical spine lateral X-ray(C), sagittal(D) and axial(E) images of post-operative T2-weighted MRI, post-operative cervical spine lateral Xray $(F)$ for 69-year-old male patient who underwent ACDF. 